\title{
Implementation and Use of SPFDs in Optimizing Boolean Networks
}

\author{
Subarnarekha Sinha Robert K. Brayton \\ Department of Electrical Engineering and Computer Sciences, \\ University of California, Berkeley, CA 94720
}

\begin{abstract}
Yamashita et. al.[1] introduced a new category for expressing the flexibility that a node can have in a multi-level network. Originally presented in the context of FPGA synthesis, the paper has wider implications which were discussed in [2]. SPFDs are essentially a set of incompletely specified functions. The increased flexibility that they offer is obtained by allowing both a node to change as well as its immediate fanins. The challenge with SPFDs is (1) to compute them in an efficient way, and (2) to use their increased flexibility in a controlled way to optimize a circuit. In this paper, we provide a complete implementation of SPFDs using BDDs and apply it to the optimization of Boolean networks. Two scenarios are presented, one which trades literals for wires and the other rewires the network by replacing one fanin at a node by a new fanin. Results on benchmark circuits are very favorable.
\end{abstract}

\section{Introduction}

At ICCAD'96, an interesting paper by Yamashita et. al. [1] was presented which develops a new way to express flexibility when implementing a node in a multi-level circuit. Classically, don't cares or incompletely specified functions (ISFs) are used to derive the flexibility at a node. These don't cares have been shown to express fully the flexibility of a node due to the noncontrollability or non-observability of a node. Satisfiability don't cares (SDCs) express that certain input patterns to a node cannot appear. Observability don't cares (ODCs) express that for certain primary input patterns, the output of a gate is not observable at any of the primary outputs. ODCs plus SDCs are equivalent to ATPG testing techniques used for redundancy removal. Since ODCs are expensive to compute, various subsets have been proposed such as CSPFs (or CODCs) introduced by Muroga. CODCs combined with image computations are implemented in SIS in the command full_simplify. CODCs can be computed relatively simply by propagating CODCs backwards through the network. At a multiple fanout point, wire CODCs on the fanouts are intersected to obtain the node CODC. Besides being computationally efficient, CODCs are independent; a CODC at one node can be used without affecting the CODC at another node. ODCs do not have this property. SPFDs are a generalization of CODCs.

To categorize different kinds of flexibility for combinational networks, Sentovich [3, 4] used three categories, ISFs, Boolean relations, and sets of Boolean relations and showed various situations where each occur. SPFDs provide a new useful category. They are essentially sets of ISFs. They can be computed efficiently and are independent, like CODCs, but express more flexibility. They are not a subset of any Boolean relation.

In this paper we define SPFDs in Section 2 and in Section
3, we give an overview on computing SPFDs and introduce some notation. In Section 4, we discuss how to compute them efficiently using BDDs. In Section 5, we provide two different methods for using them to optimize a network. Section 6 gives some experimental results and Section 7 concludes the paper and discusses future developments.

\section{SPFDs}

Definition 1 A function $f$ is said to distinguish a pair of functions $g_{1}$ and $g_{2}$ if either one of the following two conditions is satisfied:

$$
\begin{aligned}
& g_{1} \leq f \leq \bar{g}_{2} \\
& g_{2} \leq f \leq \bar{g}_{1}
\end{aligned}
$$

Note that this definition is symmetrical between $g_{1}$ and $g_{2}$. We can think of $g_{1}$ as the onset and $g_{2}$ as the offset in condition 1 or vice-versa for condition 2.

Having to satisfy only one of the two conditions provides the freedom to implement a function or its complement. This is one source of additional flexibility provided by SPFDs.

Definition 2 An SPFD

$$
\left\{\left(g_{1 a}, g_{1 b}\right), \ldots,\left(g_{n a}, g_{n b}\right)\right\}
$$

represents a Set of Pairs of Functions to be Distinguished.

Definition 3 A function $f$ satisfies an SPFD, if $f$ distinguishes each pair of the set, i.e.

$$
\begin{aligned}
& {\left[\left(\left(g_{1 a} \leq f \leq \bar{g}_{1 b}\right)+\left(g_{1 b} \leq f \leq \bar{g}_{1 a}\right)\right] \wedge \ldots \wedge\right.} \\
& {\left[\left(g_{n a} \leq f \leq \bar{g}_{n b}\right)+\left(g_{n b} \leq f \leq \bar{g}_{n a}\right)\right]}
\end{aligned}
$$

An SPFD represents flexibility that can be used to implement a node in a network - the only condition required is that the function implemented at the node satisfy its node SPFD.

A trivial case is where the set is a single pair. In this case the SPFD represents two incompletely specified functions (ISF) where one is the complement of the other. If each of the $\left\{\left(g_{1 a}, g_{1 b}\right),\left(g_{2 a},.\right), \ldots,\left(., g_{n b}\right)\right\}$ are pairwise disjoint, then the SPFD represents $2^{n}$ ISFs $^{1}$.

It is instructive to consider how to represent a given ISF. Think of each minterm $m_{i}$ in its onset as a function and each minterm $m_{j}$ in its offset as a function. Then each $\left(m_{i}, m_{j}\right)$ is a pair of functions to be distinguished, i.e. we have to find a function $f$ such that $f\left(m_{i}\right) \neq f\left(m_{j}\right)$. With ISFs it is necessary

\footnotetext{
${ }^{1}$ Note that an SPFD cannot represent a single function, it always represents at least a pair. Thus it cannot represent the function 1 .
} 
that for all $m_{i}$ in the onset, we have $f\left(m_{i}\right)=1$ and for all $m_{j}$ in the offset, we have $f\left(m_{j}\right)=0$. With SPFDs this is not imposed, just that $f$ distinguish all the pairs in the list.

This leads to the representation that we use in this paper, of an SPFD as a symmetric bipartite graph i.e. a symmetric relation $R\left(x, x^{\prime}\right.$ ) (which can be represented compactly as a BDD) where $R\left(x_{i}, x_{j}^{\prime}\right)=1 \Leftrightarrow R\left(x_{j}, x_{i}^{\prime}\right)=1$. The variable $x$ is in some space $X$. If a pair of minterms $\left(m_{i}, m_{j}\right) \in R$ (i.e. is an edge in the bipartite graph), then we seek a function $f$ such that $f\left(m_{i}\right) \neq f\left(m_{j}\right)$.

Figure 1 illustrates the bipartite graph representation of an SPFD. This shows that 000 has to be distinguished from 010 and 011 and 001 has to be distinguished from 100, but no requirements are imposed on the remaining pairs of minterms. Hence flexibility in optimizing $f$ is provided. If the bipartite

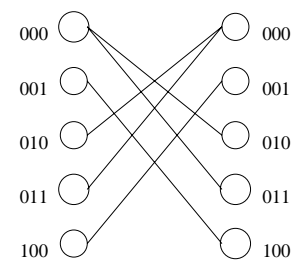

Figure 1: Bipartite graph representation of $\{(000,010),(000,011),(001,100)\}$

graph $R$ has a single strongly connected component (SCC) it is a pair of ISFs. If it has $k$ SCCs, it is a set of $2^{k}$ ISFs. In the above example, for instance, there are two SCCs which represents 4 ISFs.

Classically, in computing the flexibility at a node in a Boolean network, don't cares are computed which represent a single ISF. These computations can be generalized so that SPFDs are obtained, which provide much more freedom in optimizing the node.

\section{Overview}

\subsection{Notation}

We consider a Boolean network with primary inputs $x=$ $\left(x_{1}, \ldots, x_{n}\right)$, internal variables $y=\left(y_{1}, \ldots, y_{m}\right)$ and internal nodes $\left(\eta_{1}, \ldots, \eta_{m}\right)$. Each internal node, $\eta_{i}$, is associated with a function, $f_{i}$, a Boolean function of the variables $x, y$. The relation $y_{i}=f_{i}$ is imposed. Since the Boolean network is acyclic, each $f_{i}$ can be expressed as a global function, $g_{i}(x)$, of the primary inputs only, by recursively substituting $y_{j}=f_{j}$ until only primary inputs remain. In the sequel, we use $g_{i}(x)$ to denote $f_{i}$ expressed as a function of $x$.

The space of local input variables for $f_{i}$ is denoted $Y_{i}$ which may consist of both $x$ and $y$ variables. For example, if $f_{i}=$ $y_{3} \overline{y_{5}}+x_{6}$, then $Y_{i}=\left\{y_{3}, y_{5}, x_{6}\right\}$. The functions in the transitive fanin of $f_{i}$ define a mapping from $X$ to $Y_{i}$, denoted by $G_{i}(x)$ : $B^{n} \rightarrow B^{k}$ where $k=\left|Y_{i}\right|$.

The fanout space of node $\eta_{i}$, denoted $Z_{i}$, is the union of the input spaces of the fanouts of $i$, i.e.

$$
Z_{i}=\cup_{j \in \operatorname{fanout}_{(i)}} Y_{j}
$$

From now on, in this paper, we think of an SPFD as a symmetric bipartite graph where the vertices are minterms in some space (in the above, this is the space $Y_{i}$ ). This is represented by a relation, $R$, which is just the set of edges of the graph. In the implementation of our algorithms, each $R$ is represented by the BDD of its characteristic function.

\subsection{Computing SPFDs}

SPFDs can be computed for an entire network by starting at the primary outputs. The computation is a two-step process done in reverse topological order.

1. At each node, the edges of its SPFD are distributed to its input wires, creating wire SPFDs.

2. Once all the fanout wire SPFDs of a node are available, the node SPFD is computed as the union of the wire SPFDs.

To start, one can assume that at each primary output, a compatible don't care set is given. This translates easily into a corresponding SPFD for each output node. Suppose $o$ is a primary output and its SPFD is given as $R_{\circ}\left(X, X^{\prime}\right)$ i.e. as a bipartite graph in terms of the primary inputs ${ }^{2}$. We first map this into a SPFD $R_{o}\left(Y_{o}, Y_{o}^{\prime}\right)$ in terms of $o$ 's input space. Next each pair of minterms to be distinguished (edge) is assigned to one of the inputs wires. The set of pairs assigned to input wire $(j, o)$ is denoted $R_{j o}\left(Y_{o}, Y_{o}^{\prime}\right)$. At an internal node $\eta_{j}$ assume we have already computed its SPFD, $R_{j}\left(Y_{j}, Y_{j}^{\prime}\right)$. Then its edges are distributed to each input wire $(k, j)$ to obtain $R_{k j}\left(Y_{j}, Y_{j}^{\prime}\right)$.

Once all of the fanouts of node $\eta_{i}$ have been processed, we have $R_{i j}\left(Y_{j}, Y_{j}^{\prime}\right)$ for each wire $(i, j), j \in$ fanout $(i)$. Since the function at $\eta_{i}$ must satisfy all the $R_{i j}\left(Y_{j}, Y_{j}^{\prime}\right)$ we must have

$$
R_{i}\left(Z_{i}, Z_{i}^{\prime}\right)=\cup_{j \in \operatorname{fanout}_{(i)}} R_{i j}\left(Y_{j}, Y_{j}^{\prime}\right)
$$

The next step is to translate this into $R_{i}\left(Y_{i}, Y_{i}^{\prime}\right)$ using the mappings,

$$
G_{j}(x), j \in \operatorname{fanout}(i) \text { and } G_{i}(x)
$$

Repeating this procedure in reverse topological order from outputs to inputs we obtain SPFDs for all nodes in the network.

\subsection{Re-encoding the fanins of a function}

Consider a node $\eta_{i}$ and an associated SPFD, $R_{i}\left(Y_{i}, Y_{i}^{\prime}\right)$. Then, $f_{i}$ can be any function which distinguishes all $\left(y^{k}, y^{l}\right) \in$ $R_{i}$. Each minterm $y^{k} \in Y_{i}$ represents a function of the primary input variables $x$, i.e. $q_{y^{k}}(x)=\left\{x \mid y^{k}=G_{i}(x)\right\}$. So $R_{i}\left(Y_{i}, Y_{i}^{\prime}\right)$ induces a corresponding SPFD $R_{i}\left(X, X^{\prime}\right)$, i.e.

$$
\begin{aligned}
& R_{i}\left(X, X^{\prime}\right)= \\
& \quad\left\{\left(x^{j}, x^{p}\right) \mid x^{j} \in q_{y^{k}}, x^{p} \in q_{y^{l}},\left(y^{k}, y^{l}\right) \in R_{i}\left(Y_{i}, Y_{i}^{\prime}\right)\right\}
\end{aligned}
$$

We note that $G_{i}$ represents the current implementation of the transitive fanin of $\eta_{i}$. But we will be interested in changing $G_{i}$ (using the computed SPFDs) to improve the circuit. Any new implementation whose mapping $\hat{G}_{i}$ satisfies

$$
\hat{G}_{i}\left(x^{k}\right) \neq \hat{G}_{i}\left(x^{l}\right), \forall\left(x^{k}, x^{l}\right) \in R_{i}\left(X, X^{\prime}\right)
$$

${ }^{2}$ Usually an output don't care is given in terms of the primary inputs. 
is allowed. If the change from $G_{i}$ to $\hat{G}_{i}$ is made, $f_{i}$ must change to reflect this re-encoding. Figure 2 illustrates the change needed in $f_{i}$ where conceptually an encoding function $Y_{i}=$ $E\left(\hat{Y}_{i}\right)$ is inserted in front of $f_{i}$. The new function $\hat{f}_{i}\left(\hat{Y}_{i}\right)=$ $f_{i}\left(E\left(\hat{Y}_{i}\right)\right)$ then replaces $f_{i}$.

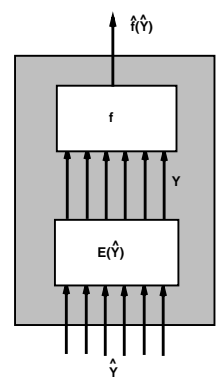

Figure 2: Function $\hat{f}$ is the original function $f$ under the new encoding of the inputs $E(\hat{Y})$

We also note that in order for a function at $\eta_{i}$ to distinguish $y^{k}$ from $y^{l}$ it is only necessary that one of its inputs has different values on these two minterms. Thus, as done in the previous section, different pairs of minterms can be assigned to different wires each of which is given the task of distinguishing the pairs assigned to it. The set of edges assigned to the input wires then constitutes a new SPFD for that wire. This may require that the function at $\eta_{i}$ be changed. Thus more flexibility is achieved, by allowing both the function and its inputs to be changed. Note also that the SPFDs generated on the wires are independent; each wire has an assigned task, and it does not matter what any other wire does as long as each satisfies its own task.

\subsection{Controlling change}

Note that in using the flexibility provided by SPFDs both the function $f_{i}$ as well as its transitive fanin may be changed, creating new functions $\hat{f}_{i}$ and a new fanin mapping, $\hat{G}_{i}(x)$. However, since it would probably be too expensive to change the entire transitive fanin of $f_{i}$, we restrict the change to only the immediate fanin of $f_{i}$ as follows.

We assign a minterm pair $\left(y^{a}, y^{b}\right) \in R_{i}\left(Y_{i}, Y_{i}^{\prime}\right)$ to a fanin $\eta_{j}$ only if

$$
g_{j}\left(x^{r}\right) \neq g_{j}\left(x^{s}\right) \forall x^{r} \in q_{y^{a}}(x), x^{s} \in q_{y^{b}}(x)
$$

In other words, $g_{j}$ must already have the power to distinguish the two minterms. This insures that only the immediate fanin may need to change to get the new encoding in the $Y_{i}$ space.

Another concern is that if a node $\eta_{i}$ is changed then it may be necessary to propagate this change through all of its transitive fanouts. In using SPFDs with LUT FPGAs, this is not really a concern, since in all the tables in the transitive fanout of an LUT, even though the function may change, the fanins to each LUT remains the same. For general Boolean networks, in the implementation that we have done so far, we have confined the propagated changes to just two nodes, $\eta_{i}$ and one of its fanins. This is done for each $\eta_{i}$. This can be done using the CODC of $i$ computed in the normal way instead of its SPFD. This is translated into an SPFD at that node, which is distributed to its inputs. One input, say $\eta_{k}$, is chosen to be changed which may imply that the function at $\eta_{i}$ must be changed, but because the CODC at the output of $\eta_{i}$ was used, no other node in its transitive fanout needs to be changed. In addition, if $\eta_{k}$ is changed, this may require other functions in the transitive fanout of $\eta_{k}$ to change. We again block this by requiring that the new function at $\eta_{k}$ satisfy the CODCs on the fanout wires of $\eta_{k}$ (other than the wire to $\eta_{i}$ ). Thus in effect, we select a region to be changed ${ }^{3}$ and surround it with a frontier of CODCs to block propagation of any changes beyond this region. Of course these restrictions limits the optimizations that can be done, and in the future, we may experiment with allowing the propagated changes to include a larger region.

\section{Computing the SPFDs of a network}

In the following, we give the resulting computations without proofs, although the reader may be persuaded of their correctness by the fact that for the benchmark circuits of Section 6, we verified the equivalence between the original and the optimized circuits.

\subsection{Computing the SPFDs at the fanin wires}

To calculate the SPFD at the fanins of a node, we assume that the SPFD, $R_{j}\left(Y_{j}, Y_{j}^{\prime}\right)$, has been computed ${ }^{4}$. Suppose we have selected an ordering on the input connections of $\eta_{j}$. We assign an edge of $R_{j}$ (a pair to be distinguished) to the first input in the ordering which distinguishes the pair. The result is that the SPFD of each fanin wire $(i, j)$ is the set of minterms that are distinguished by fanin $\eta_{i}$ but not by any of the fanin wires earlier in the ordering. Thus, the SPFD of fanin wire $(i, j)$ is

$$
\begin{aligned}
& R_{i j}\left(Y_{j}, Y_{j}^{\prime}\right)= \\
& \quad R_{j}\left(Y_{j}, Y_{j}^{\prime}\right)\left\{\prod_{k_{l} \in \operatorname{fanin}(j), k_{l}<i}\left(y_{k_{l}}=y_{k_{l}}^{\prime}\right)\right\}\left(y_{i} \neq y_{i}^{\prime}\right)
\end{aligned}
$$

The BDD $R_{i j}\left(Y_{j}, Y_{j}^{\prime}\right)$ represents the SPFD at input wire $\eta_{i}$ of node $\eta_{j}$. Each edge of $R_{j}\left(Y_{j}, Y_{j}^{\prime}\right)$ has been assigned to one of $\left\{R_{i j}\left(Y_{j}, Y_{j}^{\prime}\right)\right\}, i \in \operatorname{fanin}(j)$. The above equation is a straightforward BDD calculation.

This calculation essentially distributes the edges in the bipartite graph $R_{j}\left(Y_{j}, Y_{j}^{\prime}\right)$ among the fanins of $\eta_{j}$ so that each edge is assigned to the first fanin in the ordering that distinguishes the two vertices of the edge.

The following example illustrates how the edges are distributed. Suppose we are given a node $Z$ which has two fanins $a$ and $b$ and $a$ is before $b$ in the ordering. Let the SPFD of $Z$ be given as $\{(00,01),(00,10),(00,11)\}$. Then, the SPFDs of the wires $(a, Z)$ and $(b, Z)$ are $\{(00,10),(00,11)\}$ and $\{(00,01)\}$ respectively. Note that the edge $(00,01)$ was not assigned to $a$ because $a=0$ on both minterms. Figure 3 below shows the SPFDs of the wires $(a, Z)$ and $(b, Z)$ due to the SPFD at $Z$.

\subsection{Forming the SPFD at a node}

The SPFD at a node is obtained by the union of all the SPFDs of the fanout wires of the node. For a node $\eta_{j}$ with multiple fanout wires, the SPFD can be obtained as follows. Let

$$
Z_{j}=\cup_{i \in \operatorname{fanout}(j)} Y_{i}
$$

\footnotetext{
${ }^{3}$ In the current implementation, this is just the two nodes $\eta_{i}$ and $\eta_{k}$.

${ }^{4}$ All SPFDs are represented using BDDs.
} 


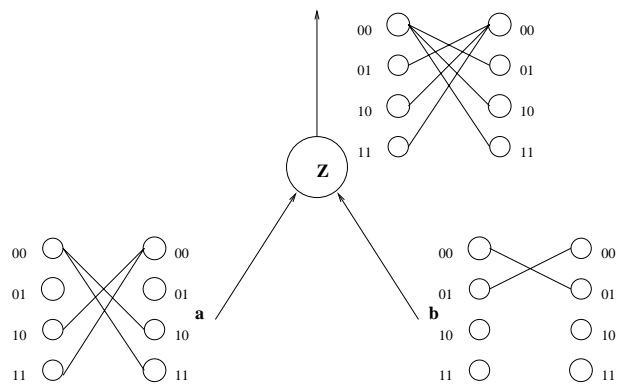

Figure 3: Computing the SPFDs of $(a, Z)$ and $(b, Z)$ from the $S P F D$ of $Z$

be the fanout space and $\mathcal{G}_{j}\left(X, Y_{j}\right)$ be the relation

$$
\mathcal{G}_{j}\left(X, Y_{j}\right)=\prod_{y_{k} \in Y_{j}}\left(y_{k}=g_{k}(x)\right)
$$

Let the SPFD associated with the $i$ th fanout wire be $R_{j i}\left(Y_{i}, Y_{i}^{\prime}\right)$. Then, the SPFD associated with $\eta_{j}$ can be obtained by taking the union of the SPFDs of the fanout wires and then taking its image in the $Y_{j}$ space. Let

$$
R_{j}\left(Z_{j}, Z_{j}^{\prime}\right)=\cup_{i \in \operatorname{fanout}_{(j)}} R_{j i}\left(Y_{i}, Y_{i}^{\prime}\right)
$$

Then the SPFD of $\eta_{j}$ is given as

$R_{j}\left(Y_{j}, Y_{j}^{\prime}\right)=\exists_{X, X^{\prime}} \mathcal{G}_{j}\left(X^{\prime}, Y_{j}^{\prime}\right) R_{j}\left(Z_{j}(X), Z_{j}^{\prime}\left(X^{\prime}\right)\right) \mathcal{G}_{j}\left(X, Y_{j}\right)$

where $R_{j}\left(Z_{j}(X), Z_{j}^{\prime}\left(X^{\prime}\right)\right)$ is obtained from $R_{j}\left(Z_{j}, Z_{j}\right)$ by composing each $z_{k} \in Z_{j}$ by $g_{k}(x)$. This technique of computing the SPFD at the output of a node in a single step may be expensive since during this computation, the entire space $\left\{Y_{j} \cup Y_{j}^{\prime} \cup X \cup X^{\prime}\right\}$ has to be considered. Moreover, the intermediate $\mathrm{BDD} R_{j}\left(Z_{j}(X), Z_{j}^{\prime}\left(X^{\prime}\right)\right)$ may be big.

An alternative approach is to map the SPFD on each fanout wire into the input space, $Y_{j}$, and then do the merging. Given the SPFD of the $i$ th fanout wire, $R_{j i}\left(Y_{i}, Y_{i}^{\prime}\right)$, its image in the local input space, $Y_{j}$, can be obtained as follows:

1. Substitute $y_{i}=g_{i}(x)$ in $R_{j i}\left(Y_{i}, Y_{i}^{\prime}\right)$ for each $y_{i} \in Y_{i}$ This gives the modified SPFD $R_{j i}\left(X, Y_{i}^{\prime}\right)$.

2. Compute $R_{j i}\left(Y_{j}, Y_{i}^{\prime}\right)=\exists_{X} \mathcal{G}_{j}\left(X, Y_{j}\right) R_{j i}\left(X, Y_{i}^{\prime}\right)$

3. Substitute $y_{i}^{\prime}=g_{i}(x)$ in $R_{j i}\left(Y_{j}, Y_{i}^{\prime}\right)$ for each $y_{i}^{\prime} \in Y_{i}^{\prime}$ to obtain the SPFD, $R_{j i}\left(Y_{j}, X\right)$.

4. Compute $R_{j i}\left(Y_{j}, Y_{j}^{\prime}\right)=\exists_{X} \mathcal{G}_{j}\left(X, Y_{j}^{\prime}\right) R_{j i}\left(Y_{j}, X\right)$

This process is repeated for the SPFDs of all the fanout wires of $\eta_{j}$. The SPFD of $\eta_{j}$, is then obtained by

$$
R_{j}\left(Y_{j}, Y_{j}^{\prime}\right)=\cup_{i \in \operatorname{fanout}(j)} R_{j i}\left(Y_{j}, Y_{j}^{\prime}\right)
$$

Note that while merging the SPFDs in the local input space, $Y_{j}$, the largest space that has be considered at any time is $\left(Y_{j} \cup Y_{j}^{\prime} \cup X\right)$, which is definitely smaller than the largest space encountered by the first method. However, note that each $R_{j i}$ is handled separately.

\subsection{Computing the function at a node}

As mentioned previously, if the fanin mapping of a node is changed, then the function at the node may have to be changed to reflect this re-encoding. We compute the SPFDs for all the nodes in the network in a reverse topological order from outputs to inputs. Then, we obtain the implementations of the functions at the nodes in topological order from inputs to outputs. So, at any step, when the function at a node is being derived, the new global functions at the input wires are already known i.e. the fanin mapping is known. To compute a set of functions that can be implemented at $\eta_{j}$, the modified SPFD of the node under the new encoding at the inputs is obtained. The new encoding $\hat{Y}_{j}$ is related to the old encoding $Y_{j}$ as:

$$
E\left(Y_{j}, \hat{Y}_{j}\right)=\exists_{X} \mathcal{G}_{j}\left(X, Y_{j}\right) \hat{\mathcal{G}}_{j}\left(X, \hat{Y}_{j}\right)
$$

The modified SPFD is then

$$
R_{j}\left(\hat{Y}_{j}, \hat{Y}_{j}^{\prime}\right)=\exists_{Y_{j}, Y_{j}^{\prime}} R_{j}\left(Y_{j}, Y_{j}^{\prime}\right) E\left(Y_{j}, \hat{Y}_{j}\right) E\left(Y_{j}^{\prime}, \hat{Y}_{j}^{\prime}\right)
$$

Figure 4 illustrates how the new encoding of the inputs changes the original SPFD.

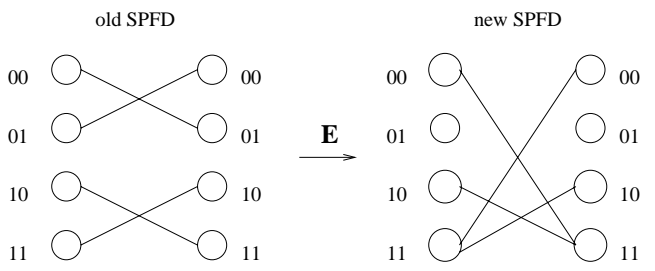

Figure 4: The modified SPFD under the encoding $E=\{00 \rightarrow$ $11,01 \rightarrow 10,10 \rightarrow 00,11 \rightarrow 11\}$

Each strongly connected component of $R_{j}\left(\hat{Y}_{j}, \hat{Y}_{j}^{\prime}\right)$ encodes the pairs of minterms that have to be distinguished. However, the minterms in one SCC do not have to be distinguished from those in another. This is similar to the graph coloring problem where any two vertices that are connected by an edge cannot be assigned the same color. In the case when $R_{j}\left(\hat{Y}_{j}, \hat{Y}_{j}^{\prime}\right)$ is bipartite, we can color all the vertices of the graph using only two colors. So, for each SCC, this corresponds to placing one set of minterms in the onset of the function and the other set in the offset. In this way, a new ISF is obtained at $\eta_{j}$. Thus, if there are $k$ strongly connected components in $R_{j}\left(\hat{Y}_{j}, \hat{Y}_{j}^{\prime}\right)$, then there are $2^{k}$ functionally different ISFs that can be implemented at $\eta_{j}$. The new implementation at a node is chosen to be the minimum of the minimum covers of all the $2^{k}$ ISFs.

\section{Non-bipartition:}

There could be situations where $R_{j}\left(\hat{Y}_{j}, \hat{Y}_{j}^{\prime}\right)$ is not bipartite, even though $R_{j}\left(Y_{j}, Y_{j}^{\prime}\right)$ is. Figure 5. illustrates one such example. In such a situation, the result is a general graph. If we can color the graph using $k$ colors, we can encode the new function using $\log k$ bits. Thus, the original node is replaced by $\log k$ nodes, all of whose fanouts are the same as the original node. This situation is undesirable since the number of fanins of the fanout nodes may increase. We are looking at ways to constrain the SPFD propagation through the network so that under any encoding, the graph $R_{j}\left(\hat{Y}_{j}, \hat{Y}_{j}^{\prime}\right)$ remains bipartite. 


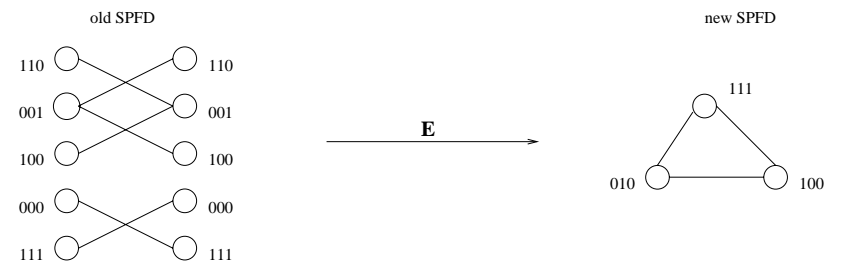

Figure 5: The modified SPFD under the encoding $E=\{110 \rightarrow$ $111,101 \rightarrow 010,001 \rightarrow 100,000 \rightarrow 010,111 \rightarrow 111\}$

It should be emphasized that in both cases all the minterms in any SCC of the original SPFD are still distinguished. But the new encoding can cause two SCCs of the original SPFD to become incompatible and hence destroy the bipartite structure. This situation can only arise if the SPFD at a node has more than one strongly connected component. Our intial experience is that non-bipartite structures occur rarely. In the experiments described in Section 5, this cannot happen since there is only one SCC at each node.

\section{Finding the SCCs:}

Given an SPFD, $R\left(z, z^{\prime}\right)$, the individual SCCs can be obtained as follows. Initially, the two step graph $R_{2}\left(z, z^{\prime}\right)=$ $\exists_{y} R(z, y) R\left(y, z^{\prime}\right)$ and the set of left-side nodes $N(z)=$ $\exists_{y} R(z, y)$ in the bipartite graph are obtained. Then the following steps are performed.

1. Pick $z_{0} \in N(z)$.

2. Compute the fixpoint, $E_{1}(z)$, which is all the nodes that can be reached from $z_{0}$ using $R_{2}$. Compute $E_{0}(z)=$ $\exists_{y} R(y, z) E_{1}(y)$, the set of nodes that are connected by an edge to a node in $E_{1}(z)$. Store $\left(E_{1}, E_{0}\right)$ as an SCC pair.

3. Let $N(z)=N(z) \overline{E_{1}(z)+E_{0}(z)}$. If $N \neq \emptyset$, go to 1 .

\section{Circuit Optimization using SPFDs}

\subsection{Minimum fanin computation}

The objective here is given a node $\eta_{j}$ determine if any of the fanin wires can be removed, or if not can any of the fanins be simplified enough to obtain an improved cost function.

In order to simplify a fanin, $\eta_{k}$, as much as possible, we compute the minterms that are distinguished uniquely by that fanin. The minimum SPFD of the wire $(k, j), R_{k j}^{m i n}$ gives the minterms that need to be distinguished at the output of $\eta_{j}$ and can be done only by the wire $(k, j)$. Given the SPFD, $R_{j}\left(Y_{j}, Y_{j}^{\prime}\right)$, of $\eta_{j}$,

$$
R_{k j}^{m i n}\left(Y_{j}, Y_{j}^{\prime}\right)=R_{j}\left(Y_{j}, Y_{j}^{\prime}\right)\left\{\prod_{y_{i} \in Y_{j}, i \neq k}\left(y_{i}=y_{i}^{\prime}\right)\right\}\left(y_{k} \neq y_{k}^{\prime}\right)
$$

If $R_{k j}^{\min }$ is nil, we could remove the wire, but we do not do this until it is determined that the new function at $\eta_{j}$ is small enough. If $R_{k j}^{\min } \neq \phi$, then a new function is obtained at $\eta_{k}$ that satisfies $R_{k j}^{\min }$. We then test if the result also satisfies the CODCs of all the other fanouts of $\eta_{k}$. If so, a new encoding at the inputs of $\eta_{j}$ due to the new function at $\eta_{k}$ is computed. This is related to the old encoding $Y_{j}$ as:

$$
E\left(Y_{j}, \hat{Y}_{j}\right)=\exists_{X} \mathcal{G}_{j}\left(X, Y_{j}\right) \hat{\mathcal{G}}_{j}\left(X, \hat{Y}_{j}\right)
$$

Note that if $R_{k j}^{\text {min }}=\phi$, wire $(k, j)$ can be removed. This is equivalent to setting the term $\hat{y}_{k}=g_{k}(x)$ to 1 in the computation of $\hat{\mathcal{G}}_{j}\left(X, \hat{Y}_{j}\right)$.

The modified SPFD of $\eta_{j}$ under the new encoding, $E\left(Y_{j}, \hat{Y}_{j}\right)$, is

$$
R_{j}\left(\hat{Y}_{j}, \hat{Y}_{j}^{\prime}\right)=\exists_{Y_{j}, Y_{j}^{\prime}} R_{j}\left(Y_{j}, Y_{j}^{\prime}\right) E\left(Y_{j}, \hat{Y}_{j}\right) E\left(Y_{j}^{\prime}, \hat{Y}_{j}^{\prime}\right)
$$

which is minimized as discussed in the previous section. The gain of these changes is computed as

$$
\begin{aligned}
\operatorname{gain}_{k j}= & l_{n}^{\text {old }}+l_{f}^{\text {old }} \Leftrightarrow\left\{l_{n}^{\text {new }}+l_{f}^{\text {new }}\right\} \\
& +\alpha *\left\{w_{n}^{\text {old }}+w_{f}^{\text {old }} \Leftrightarrow\left\{w_{n}^{n e w}+w_{f}^{n e w}\right\}\right\}
\end{aligned}
$$

where $l$ stands for the literal count in the factored form, $w$ the number of fanins of the node, the superscripts, old and new, refer to the original and new implementations respectively and the subscripts, $n$ and $f$, refer to the node and fanin respectively. The parameter $\alpha$ above relates the value of a wire relative to a literal.

Since it is not practical to do this computation for each fanin wire of $\eta_{j}$, in our current experiments, only a subset of the fanins are considered; only those fanins whose own fanins are a subset of the fanins of $\eta_{j}$ are selected. This procedure is repeated for each candidate fanin and the one giving the largest non-negative gain, gain $_{k j}$, is chosen. Then the original function at $\eta_{j}$ and the corresponding fanin function are replaced by the new ones. This procedure of trying to simplify the fanin of a node as much as possible is called fanin_simplify.

\subsection{Alternative wire computation}

The objective here is to replace wire $(k, j)$ to node $\eta_{j}$ with a wire from another node $\eta_{s}$, originally not a fanin of node $\eta_{j}$, such that a new function $\hat{f}_{j}$ can be found which depends on $\eta_{s}$ but not on $\eta_{k}$. The function $\hat{f}_{j}$ must still satisfy the SPFD at $\eta_{j}$ and some gain should be obtained by this replacement.

A wire $(s, j)$ can replace the wire $(k, j)$ if all the minterms in $m_{i} \in X$ uniquely distinguished by the wire $(k, j)$ are also distinguished by $\eta_{s}$. The procedure for replacing one wire by another is explained below.

Suppose $R_{j}\left(Y_{j}, Y_{j}^{\prime}\right)$ has been computed. The minimum SPFD of the wire, $(k, j)$, is computed. Now we seek candidate nodes $\left\{\eta_{s}\right\}$ in the network that can distinguish all the minterms in $R_{k j}^{\text {min }}\left(Y_{j}, Y_{j}^{\prime}\right)$. A necessary and sufficient condition for this is that $H\left(y_{s}\right)=\phi$, where $H\left(y_{s}\right)$ is derived by the following steps.

1. Substitute $y_{j}=g_{j}(x)$ in $R_{k j}^{\min }\left(Y_{j}, Y_{j}^{\prime}\right)$ for each $y_{j} \in Y_{j}$ to obtain $R_{k j}^{\min }\left(x, Y_{j}^{\prime}\right)$.

2. Compute

$R_{k j}^{\min }\left(y_{s}, Y_{j}^{\prime}\right)=\left\{\exists_{x}\left(y_{s}=g_{s}(x)\right) R_{k j}^{\min }\left(x, Y_{j}^{\prime}\right)\right\}$.

3. Substitute $y_{j}^{\prime}=g_{j}(x)$ in $R_{k j}^{\min }\left(y_{s}, Y_{j}^{\prime}\right)$ for each $y_{j}^{\prime} \in Y_{j}^{\prime}$ to obtain $R_{k j}^{\min }\left(y_{s}, x\right)$. 
4. Compute $H\left(y_{s}\right)=\left\{\exists_{x}\left(y_{s}=g_{s}(x)\right) R_{k j}^{\min }\left(y_{s}, x\right)\right\}$.

$H\left(y_{s}\right)$ has the property that if $H\left(y_{s}\right) \neq \emptyset$, then there exists at least one pair of minterms in $R_{k j}^{\min }\left(Y_{j}, Y_{j}^{\prime}\right)$ that are not distinguished by $\eta_{s}$ and hence $\eta_{s}$ cannot be a candidate.

Since it is not practical to consider all the nodes in the network, only a subset is considered; only the fanins of $\eta_{k}$ and the nodes in their transitive fanout are considered. Of course, nodes in the transitive fanout of $\eta_{j}$ cannot be considered.

After the set of candidate nodes is obtained, the following procedure is repeated to obtain the node $\eta_{s}$ from which a wire can be added that can replace wire $(k, j)$. First, the modified SPFD of $\eta_{j}$ is obtained in the new space

$$
\hat{Y_{j}}=\left\{y_{i} \in Y_{j}, i \neq k\right\} \cup\left\{y_{s}\right\}
$$

A new minimized function at node $\eta_{j}$ is then obtained from this modified SPFD as previously described. If the number of literals in the factored form of the new function is less than the number in the factored form of $f_{j}$, the replacement is done $e^{5}$. In case of a tie in the number of literals, the replacement is also done if the level of $\eta_{s}$ is less than the level of $\eta_{k}$. Otherwise, the next node in the candidate set is selected and the same procedure repeated. We call this procedure of replacing wires by other wires, wire_replace.

To illustrate the power of wire_replace, consider

$$
\begin{aligned}
& z=\bar{g} b+g \bar{b} \\
& g=\bar{a} b+a \bar{b}
\end{aligned}
$$

Running full_simplify on this example results in no simplification. Now consider wire_replace. Wires $(a, g)$ and $(b, g)$ have no alternative wires and hence $g$ remains unchanged. For wire $(g, z)$, the minimum SPFD is $A=\{(00,10),(01,11)\}$. (In the set $A$, each minterm is of the form $g b$ ). Now, if we express the minterms of $A$ in terms of the primary inputs, $a$ and $b$, we get $A^{\prime}=\{(00,10),(11,01)\}$. (The minterms in $A^{\prime}$ are of the form $a b)$. The primary input, $a$, can distinguish both pairs in $A^{\prime}$. Hence, $a$ is a candidate node and can be used to replace $(g, z)$. Simplifying $z$ we obtain $z=a$. It is also interesting to note that in $g,\{00,11\}$ are in the offset and $\{01,10\}$ are in the onset. However for $a,\{00,01\}$ are in the offset and $\{11,10\}$ are in the onset. Yet, $a$ can be used to replace $g$. This ability to mix onset and offset minterms is another source of additional flexibility provided by SPFDs.

\section{Results}

The results for fanin simplification are shown in Table 1 . The initial circuits were obtained from the various benchmarks by running script.rugged in SIS on them. The result served as our point of comparison. We then took this output and subjected it to an iteration of fanin_simplify until no gain was obtained or the cost function (which was equal to the gain in the number of literals plus twice the gain in the number of wires) became non-positive for the first time. In some circuits, the cost function kept oscillating and for these circuits we took the point at which the cost function first started to oscillate. We recorded, under the heading, (fanin_simplify)*, both the number of connections in the circuit "wires" and the number of

\footnotetext{
${ }^{5}$ Of course, we could use the gain function given in Section 5.1 to control acceptance, but the experiment here is to reduce literals (and hopefully wires too).
}

\begin{tabular}{|c|c|c|c|c|c|c|}
\hline \hline & \multicolumn{2}{|c|}{ script.rugged } & \multicolumn{4}{|c|}{ (fanin_simplify $)^{*}$} \\
\hline NAMES & wires & literals & wires & ratio & literals & ratio \\
apex6 & 650 & 741 & 633 & 0.974 & 731 & 0.987 \\
apex7 & 222 & 245 & 210 & 0.946 & 241 & 0.984 \\
b9 & 115 & 122 & 111 & 0.965 & 122 & 1 \\
bbara & 51 & 63 & 45 & 0.882 & 61 & 0.968 \\
bbse & 140 & 140 & 138 & 0.986 & 140 & 1 \\
c1908 & 378 & 540 & 357 & 0.944 & 534 & 0.989 \\
c432 & 205 & 205 & 194 & 0.946 & 201 & 0.98 \\
c499 & 344 & 552 & 296 & 0.86 & 554 & 1.004 \\
c8 & 128 & 139 & 118 & 0.922 & 144 & 1.036 \\
cht & 165 & 165 & 164 & 0.994 & 165 & 1 \\
cse & 213 & 215 & 201 & 0.944 & 206 & 0.958 \\
dk16 & 348 & 348 & 307 & 0.882 & 345 & 0.991 \\
dk17 & 88 & 89 & 44 & 0.5 & 58 & 0.652 \\
ex1 & 279 & 280 & 259 & 0.928 & 261 & 0.932 \\
ex2 & 172 & 172 & 158 & 0.919 & 173 & 1.006 \\
ex3 & 84 & 86 & 81 & 0.964 & 87 & 1.012 \\
ex4 & 91 & 91 & 87 & 0.956 & 87 & 0.956 \\
ex5 & 71 & 71 & 47 & 0.662 & 66 & 0.93 \\
ex6 & 108 & 109 & 96 & 0.889 & 111 & 1.018 \\
f51m & 60 & 91 & 39 & 0.65 & 88 & 0.967 \\
frg1 & 79 & 136 & 60 & 0.759 & 137 & 1.007 \\
frg2 & 833 & 886 & 753 & 0.904 & 894 & 1.009 \\
i6 & 391 & 457 & 391 & 1 & 457 & 1 \\
i7 & 587 & 596 & 517 & 0.881 & 583 & 0.978 \\
i8 & 1012 & 1015 & 989 & 0.977 & 999 & 0.984 \\
i9 & 587 & 596 & 577 & 0.983 & 603 & 1.012 \\
k2 & 1112 & 1120 & 1084 & 0.975 & 1100 & 0.982 \\
kirkman & 300 & 308 & 87 & 0.29 & 135 & 0.438 \\
lal & 89 & 105 & 83 & 0.933 & 103 & 0.981 \\
planet & 614 & 617 & 594 & 0.967 & 610 & 0.989 \\
s1 & 429 & 430 & 356 & 0.83 & 375 & 0.872 \\
sand & 612 & 613 & 579 & 0.946 & 584 & 0.953 \\
scf & 983 & 985 & 974 & 0.991 & 977 & 0.992 \\
sct & 63 & 79 & 55 & 0.873 & 78 & 0.987 \\
sse & 140 & 140 & 109 & 0.779 & 117 & 0.836 \\
styr & 596 & 596 & 561 & 0.941 & 565 & 0.948 \\
term1 & 130 & 179 & 104 & 0.8 & 174 & 0.972 \\
too_large & 266 & 347 & 255 & 0.959 & 349 & 1.006 \\
tt2 & 184 & 219 & 167 & 0.908 & 215 & 0.982 \\
vda & 611 & 615 & 606 & 0.992 & 612 & 0.995 \\
x1 & 285 & 298 & 275 & 0.965 & 301 & 1.01 \\
x2 & 44 & 48 & 42 & 0.955 & 49 & 1.021 \\
x3 & 720 & 787 & 675 & 0.938 & 764 & 0.971 \\
x4 & 367 & 386 & 353 & 0.962 & 384 & 0.995 \\
AVERAGE & 29 & 41 & 20 & 0.69 & 41 & 1 \\
\hline \hline & & & 0.887 & & 0.962 \\
\hline
\end{tabular}

Table 1: Results for fanin_simplify 


\begin{tabular}{|c|c|c|c|c|c|c|c|c|c|c|}
\hline & \multicolumn{2}{|c|}{ script.rugged } & \multicolumn{4}{|c|}{ (wire_replace)* } & \multicolumn{4}{|c|}{ (script.rugged, (wire_replace)*)* } \\
\hline NAMES & wires & literals & wires & ratio & literals & ratio & wires & ratio & literals & ratio \\
\hline apex6 & 650 & 741 & 625 & 0.962 & 724 & 0.977 & 621 & 0.955 & 715 & 0.965 \\
\hline apex 7 & 222 & 245 & 203 & 0.914 & 235 & 0.959 & 198 & 0.892 & 226 & 0.922 \\
\hline b9 & 115 & 122 & 111 & 0.965 & 119 & 0.975 & 112 & 0.974 & 122 & 1 \\
\hline bbara & 51 & 63 & 46 & 0.902 & 60 & 0.952 & 49 & 0.961 & 61 & 0.968 \\
\hline bbsse & 140 & 140 & 119 & 0.85 & 126 & 0.9 & 102 & 0.729 & 110 & 0.786 \\
\hline c1908 & 378 & 540 & 352 & 0.931 & 525 & 0.972 & 352 & 0.931 & 525 & 0.972 \\
\hline $\mathrm{c} 432$ & 205 & 205 & 186 & 0.907 & 222 & 1.083 & 186 & 0.907 & 222 & 1.083 \\
\hline c499 & 344 & 552 & 300 & 0.872 & 552 & 1 & 300 & 0.872 & 552 & 1 \\
\hline c8 & 128 & 139 & 127 & 0.992 & 138 & 0.993 & 125 & 0.977 & 136 & 0.978 \\
\hline cht & 165 & 165 & 164 & 0.994 & 165 & 1 & 163 & 0.988 & 164 & 0.994 \\
\hline cse & 213 & 215 & 201 & 0.944 & 204 & 0.949 & 162 & 0.761 & 183 & 0.851 \\
\hline $\mathrm{dk} 16$ & 348 & 348 & 316 & 0.908 & 321 & 0.922 & 187 & 0.537 & 245 & 0.704 \\
\hline $\mathrm{dk} 17$ & 88 & 89 & 40 & 0.455 & 53 & 0.596 & 37 & 0.42 & 51 & 0.573 \\
\hline ex 1 & 279 & 280 & 255 & 0.914 & 258 & 0.921 & 219 & 0.785 & 229 & 0.818 \\
\hline ex2 & 172 & 172 & 142 & 0.826 & 160 & 0.93 & 134 & 0.779 & 151 & 0.878 \\
\hline ex3 & 84 & 86 & 82 & 0.976 & 85 & 0.988 & 45 & 0.536 & 62 & 0.721 \\
\hline ex4 & 91 & 91 & 82 & 0.901 & 85 & 0.934 & 71 & 0.78 & 78 & 0.857 \\
\hline ex 5 & 71 & 71 & 60 & 0.845 & 67 & 0.944 & 26 & 0.366 & 51 & 0.718 \\
\hline ex6 & 108 & 109 & 92 & 0.852 & 103 & 0.945 & 89 & 0.824 & 96 & 0.881 \\
\hline $\mathrm{f} 51 \mathrm{~m}$ & 60 & 91 & 39 & 0.65 & 83 & 0.912 & 45 & 0.75 & 70 & 0.769 \\
\hline frg 1 & 79 & 136 & 44 & 0.557 & 127 & 0.934 & 51 & 0.646 & 127 & 0.934 \\
\hline frg 2 & 833 & 886 & 696 & 0.836 & 792 & 0.894 & 690 & 0.828 & 735 & 0.83 \\
\hline i6 & 391 & 457 & 391 & 1 & 457 & 1 & 391 & 1 & 457 & 1 \\
\hline i7 & 518 & 584 & 517 & 0.998 & 583 & 0.998 & 517 & 0.998 & 583 & 0.998 \\
\hline i8 & 1012 & 1015 & 980 & 0.968 & $988 *$ & $0.973^{*}$ & & & & \\
\hline i9 & 587 & 596 & 584 & 0.995 & 596 & 1 & 580 & 0.988 & 592 & 0.993 \\
\hline $\mathrm{k} 2$ & 1112 & 1120 & 1067 & 0.96 & 1082 & 0.966 & $*$ & & $*$ & \\
\hline kirkman & 300 & 308 & 137 & 0.457 & 198 & 0.643 & 85 & 0.283 & 126 & 0.409 \\
\hline lal & 89 & 105 & 82 & 0.921 & 101 & 0.962 & 79 & 0.888 & 102 & 0.971 \\
\hline planet & 614 & 617 & 586 & 0.954 & 593 & 0.961 & 555 & 0.904 & 589 & 0.955 \\
\hline s1 & 429 & 430 & 349 & 0.814 & 381 & 0.886 & 275 & 0.641 & 298 & 0.693 \\
\hline sand & 612 & 613 & 566 & 0.925 & 574 & 0.936 & 521 & 0.851 & 550 & 0.897 \\
\hline scf & 983 & 985 & 970 & 0.987 & 974 & 0.989 & 870 & 0.885 & 917 & 0.931 \\
\hline sct & 63 & 79 & 57 & 0.905 & 78 & 0.987 & 55 & 0.873 & 75 & 0.949 \\
\hline sse & 140 & 140 & 119 & 0.85 & 126 & 0.9 & 102 & 0.729 & 110 & 0.786 \\
\hline styr & 596 & 596 & 550 & 0.923 & 555 & 0.931 & 431 & 0.723 & 482 & 0.809 \\
\hline term 1 & 130 & 179 & 97 & 0.746 & 152 & 0.849 & 93 & 0.715 & 103 & 0.575 \\
\hline too_large & 266 & 347 & 253 & 0.951 & 234 & 0.674 & $*$ & & $*$ & \\
\hline $\mathrm{ttt} 2$ & 184 & 219 & 160 & 0.87 & 206 & 0.941 & 122 & 0.663 & 163 & 0.744 \\
\hline vda & 611 & 615 & 607 & 0.993 & 612 & 0.995 & 571 & 0.935 & 579 & 0.941 \\
\hline $\mathrm{x} 1$ & 285 & 298 & 279 & 0.979 & 295 & 0.99 & 279 & 0.979 & 295 & 0.99 \\
\hline $\mathrm{x} 2$ & 44 & 48 & 43 & 0.977 & 48 & 1 & 39 & 0.886 & 46 & 0.958 \\
\hline $\mathrm{x} 3$ & 720 & 787 & 650 & 0.903 & 753 & 0.957 & 628 & 0.872 & 705 & 0.896 \\
\hline $\mathrm{x} 4$ & 367 & 386 & 347 & 0.946 & 381 & 0.987 & 332 & 0.905 & 367 & 0.951 \\
\hline $\mathrm{z} 4 \mathrm{ml}$ & 29 & 41 & 28 & 0.966 & 38 & 0.927 & 28 & 0.966 & 38 & 0.927 \\
\hline AVERAGE & & & & 0.888 & & 0.936 & & 0.807 & & 0.871 \\
\hline
\end{tabular}

Table 2: Results for wire_replace 
literals in the factored forms of the Boolean network. The ratio of these results to the output of script.rugged is also shown. At the bottom of the table we compute the average ratios for both the wires and the literals. We see, on average, a $11 \%$ reduction in the number of wires as well as a $3 \%$ reduction in literals. But since we assigned greater weights to wires $(\alpha=2)$, there is a greater reduction in wire count.

The results for wire replacement are shown in the Table 2 . The initial circuits were obtained as in the previous case. We took the output of script.rugged and subjected it to an iteration of wire_replace until no gain was obtained. The number of wires, the number of literals in the factored form of the network and the ratio of these results to the output of script.rugged were stored under the heading (wire_replace)*. For $i 8$, the program ran out of memory before the iterations could converge, so we took the values of the previous iteration. The third set of columns was obtained by taking the result of (wire_replace)* and repeating script.rugged followed by (wire_replace)* until no gain was recorded. For $k 2$ and toolarge, the program ran out of memory even before the first iteration was over. At the bottom of the table we compute the average ratios for both experiments and for both wires and for literals. We see on average a $11 \%$ reduction in wires and $6 \%$ in literals after (wire_replace)*, and still better results for the repetition of script.rugged and (wire_replace)*, a 19\% reduction in wires and $12 \%$ in literals.

The experiments were made with automation in mind. We wanted to devise an automatic script that could be run on any module without having to interact with it. The results are the "scripts", (script.rugged(fanin_simplify)*) and (script.rugged(wire_replace)*)*.

In (script.rugged (fanin_simplify)*), it is possible to trade off wires for literals or vice-versa by setting different costs to the literals and wires. In (script.rugged (wire_replace)*)*, we could set conditions for choosing an alternative wire for a given wire. In addition to minimizing literals, we could use this to optimize different criteria like delay, maximum wire length, etc.

Since the computation at a node can take an arbitrarily long time, we have introduced timeouts to prevent the computation from hanging up. So, with every node in the network we associate a timeout interval and if the time of computation exceeds the interval, we just quit that node and move on to the next. Right now, we set a timeout interval of $20 \mathrm{sec}$., more for ruggedizing the algorithms than a concern for the run time. We are looking at a number of techniques to improve the run times.

In the future, we see this technology as important for improving delay and wireability for DSM designs.

\section{Conclusions}

We have implemented the computation of SPFDs using BDDs and have shown that their use is quite feasible in circuits of medium size. Roughly, any circuits where script.rugged can be used, SPFDs can also be used. Ultimately, both fanin_simplify and wire_replace can be made more rugged following suggestions by Savoj [5], by filtering out known problems and by better controlling time-outs on some of the BDD calculations. The initial experiments with fanin_simplify and wire_replace are very encouraging. In these experiments, we have strongly restricted the SPFDs computed, in order to control the change in the network. We want to experiment with relaxing these conditions while still controlling the gains made in a predictable way.
Ultimately, we plan to use SPFDs in DSM synthesis where the ability to assign costs to the wires during circuit optimization or to replace one wire by another should be very useful to alleviate wiring problems or to improve wire delay and noise problems.

\section{Acknowledgements}

This research was supported by the SRC under contract DC324, and the California MICRO program with participating industrial sponsors, Cadence, Motorola, and Fujitsu.

\section{References}

[1] S. Yamashita, H. Sawada, and A. Nagoya. A New Method to Express Functional Permissibilities for LUT based FPGAs and Its Applications. In International Conference on Computer Aided Design, pages 254-261, November 1996.

[2] Robert K. Brayton. Understanding SPFDs: A New Method for Specifying Flexibility. In IWLS, 1997.

[3] E. M. Sentovich and R. K. Brayton. Multiple Boolean Relations. In International Workshop on Logic Synthesis, Tahoe City, CA, May 1993.

[4] Ellen M. Sentovich, Vigyan Singhal, and Robert K. Brayton. Boolean Function Sets. In unpublished manuscript, 1996.

[5] H. Savoj. Improvements in Technology Independent Optimization of Logic Circuits. In IWLS, 1997. 